\title{
Whys and Wherefores of Open Aperture ESTEM for in-situ Catalyst Reaction Studies
}

Edward D Boyes ${ }^{1,3}$ and Pratibha L Gai ${ }^{1,2,3}$

${ }^{1 .}$ Depts of Physics, ${ }^{2 .}$ Chemistry, and ${ }^{3 .}$ York JEOL Nanocentre, University of York, UK.

Heterogeneous solid-state catalysts for gas reaction chemistry, often with nanoparticle or single atom active phases highly dispersed on ceramic supports, are widely used in chemical industry manufacturing and for environmental controls. Catalysis contributes to $>25 \%$ of GDP as well as to societal well-being. The goal of ESTEM is single atom resolution in-situ studies of continuous dynamic chemical reaction processes under realistic conditions of controlled gas atmosphere and specimen temperature. It accesses key intermediate reaction states which may only exist in dynamic in-situ experiments with continuous gas flow, because they may be metastable with respect to reaction conditions of temperature or gas environment. They cannot therefore be studied reliably ex-situ. Many reactive species have to be restored or activated in the reactor after transfer through air, as is common practice industrially, and we need to do something similar to achieve real world relevant results. Catalyst surfaces deactivate chemically and by single atom and small particle migration, leading to the growth of larger entities with a lower fraction of surface atoms accessible for active reaction (the bang-for-buck criterion); and even fewer in favoured low co-ordination selective sites. The mechanisms and dynamics of the underlying deactivation processes, atom-by-atom, determine reaction outcomes and thereby the feasibility and costs of commercial reactor processes. ESTEM analyses of single atoms attached to nanoparticle surfaces and existing independently guide better operational practices in existing processes and new developments.

There is a long history of environmental facilities on older TEMs, reviewed by Butler and Hale [1], which took off scientifically at modest (nms) resolution with the HVEMs of the 1970s (Swann [2], Gai [3], and in contemporaneous TEM projects. HR ETEM came in with the purpose-built E-conversion of a then state-of-the-art Phillips CM30 HRTEM in the early 1990s (at DuPont Co, USA, Boyes and Gai [4]). The approach was based on the then innovative and now standard multiple differentially pumped ECELL apertures up and down the microscope column; starting inside the tip of each objective lens polepiece, rather than in the gap; and adding new gas tolerant TMP pumping systems while retaining resolution. In this way the highest pressure gas region is restricted to a few $\mathrm{mm}$ of beam path length and with sub-mbar or low mbar gas pressures does not affect resolution. This design is very successful for TEM, producing 100s of publications from 20 sites globally using commercial instruments based on the DuPont design. More recent versions benefit from TEM image aberration correction. This is especially important for dynamic in-situ experiments where image interpretation often needs to be based on single images (at optimum, near zero, defocus) of ever changing scenes, restricting use of the usual through focal series, and to enable high contrast TEM imaging with small amounts of negative Cs [5].

A revised approach is needed to support full STEM imaging and analysis, with the gas control aperture arrangements re-designed to support a full range of signals. For optimum results a probe corrector, not normally fitted to an ETEM, is required. A combined ESTEM and ETEM system with both probe and image correctors has been built in-house at York on a JEOL 2200 FS platform, with full performance of the core retained uncompromised and in parts enhanced. Full HAADF STEM Z-contrast imaging with single atom resolution and sensitivity is enabled, as is high quality EDX analysis (now enhanced rather than diminished by additional column apertures), and introduces key new mapping functionalities [6]. Even with our early (2006) platform system at York, we have sub-Angstrom $(<0.1 \mathrm{~nm})$ imaging in both ETEM and ESTEM modes. Furnace holder electronics must be improved for ESTEM resolution [6] but 
the introduction of high stability MEMS hot stages (here by DENS Solutions) have greatly improved diverse applications. Short mark (exposure) to long space (beam blanked) ratios can now be used to analyse and limit e-beam effects which could otherwise lead to chemical artefact sampling.

The main ESTEM competition, and vice versa, is specimen holders with gas containment windows, after Cremer [7] in modern form and earlier from JEOL. They can sustain higher gas pressures, into the bar range, but not the $>100$ bar which may be used in industry. Both the entrance e-beam illumination and exit signals having to pass through relatively thick windows (each, $>20 \mathrm{~nm}$ nominal) of a variant of $\mathrm{Si}_{3} \mathrm{~N}_{4}$ as well as up to $100 \mu \mathrm{m}$ of gas (calculations suggest this is serious if heavier than $\mathrm{H}_{2}$ ) between bulging windows. It is reported all the signals inevitably have evidence of the windows, while in the open aperture system the beam path can be made clear, or with a very thin support film. With the open aperture 'gas-in-microscope' system, a variety of existing specimen holders can all be used because the very efficient gas supply to the specimen is separate from the holder. The 'open' holders can have more functions, including double tilt heating for crystallography and for tomography. In many cases the open aperture system will also have microscopy performance and background vacuum advantages not available for 'gas-in-holder' solutions. For traditional $3 \mathrm{~mm}$ discs or complete grids, furnace holders remain essential and a major advantage of the open aperture ESTEM (and ETEM) ECELL approach. In our ESTEM we can use most existing types of specimen holder already available in the lab, at zero additional cost and short set-up times, whereas holders with windows are made in only a few designs, restricted both technically and commercially, and new types may have long lead times and high costs.

The Pa-mbar gas pressures currently sustained in the first open aperture full function ESTEM/ETEM are delivered continuously and orders of magnitude higher than the often discontinuous supply in many surface science experiments. Somorjai et al (2007) [8] have defined 'high pressure' for surface reactions as being $>0.001$ torr, or in other terms $\sim 1000 \mathrm{~mL} / \mathrm{sec}$ of gas supply, and this is only $1 \%$ of the $10 \mathrm{~Pa}$ (and $100,000 \mathrm{~mL} / \mathrm{sec}$ ) routinely used in the ESTEM, where $>99 \%$ of gas molecules are surface incident. There is actually an alternative argument for using in EM minimum gas pressures to ensure the desired chemical outcome because any excess may add to the potential for artefacts from e-beam ionised gas. In a recent paper we have shown that in repeated $\mathrm{Cu}_{2} \mathrm{O} / \mathrm{Cu}$ Redox reactions the ESTEM system supports the gas pressures needed to drive sequences of reduction and oxidation [9]. These are fully reversible with changes in gas type, in this case hydrogen and oxygen, and the endpoint structures produced are the same as in ex-situ reactions at 1 bar pressure, but of course in the ESTEM we benefit from atom-byatom (in the $\mathrm{Cu}_{2} \mathrm{O} / \mathrm{Cu}$ case in columns) details of the mechanisms to guide process optimisation [10].

References:

[1] E P Butler, K F Hale, 'Dynamic Experiments in the Electron Microscope', North Holland, (1981)

[2] P R Swann and N J Tighe, Jernkont Ann, 155 (1971), p. 497.

[3] P L Gai, Philosophical Magazine, 43 (19810), p. 841.

[4] E D Boyes and P L Gai, Ultramicroscopy, 67 (1997), p. 219.

[5] C L Jia et al, Ultramicroscopy, 110 (2010), p. 500.

[6] E D Boyes et al, Annalen der Physik, 525 (2013), p. 423.

[7] J Cremer et al, Ultramicroscopy, 108 (2008), p. 993.

[8] G A Somorjai, R L York, D Butcher and J Y Park, Phys. Chem. Chem. Phys, 9 (2007), p. 3500. [9] A P LaGrow et al, J Am Chem Soc, 139 (2017), p. 179.

[10] The University of York and EU European Regional Development Fund are acknowledged for funding the core AC machine and the EPSRC (UK) for grant EP/J018058/1 for development, staff support and initial applications of the York ESTEM. 